\title{
When a patient dies by suicide: incidence, implications and coping strategies
}

\author{
Sharon R. Foley \& Brendan D. Kelly
}

\begin{abstract}
Patient suicide is a relatively common event: $50-70 \%$ of consultant psychiatrists and $40-50 \%$ of psychiatric trainees have experienced at least one patient suicide. Patient suicide can have significant personal and professional effects on psychiatrists, including increased stress, social withdrawal, disruption to relationships, symptoms of post-traumatic stress disorder and consideration of early retirement. Following patient suicide, psychiatrists derive most support from informal contacts with team members, family and friends; additional support may be available through formal support structures or local team initiatives. It is important to place appropriate emphasis on the effects of patient suicide in psychiatry training programmes; to maintain awareness of the importance of informal supports following patient suicide; to strengthen formal systems of support; to identify other potential forms of assistance (e.g. spiritual support); and to develop local team initiatives in this area.
\end{abstract}

At some point in their careers, most mental health workers will lose a patient to suicide during the course of treatment. This event, which we call a 'patient suicide' here, is a relatively common experience among psychiatrists and could reasonably be regarded as an occupational hazard. In spite of these facts, the effects of patient suicide on mental health workers are rarely discussed and research literature on this topic is relatively sparse. In addition, there is no formal training available to many mental health workers to prepare them for the personal and professional effects of patient suicide.

In this article we outline existing knowledge about the incidence of the experience of patient suicide among mental health workers, especially psychiatrists, its effects on them and mechanisms used to cope with it. We also briefly describe a pilot scheme for dealing with patient suicide in one community mental health team and make recommendations for future developments in this area.

\section{Incidence}

A limited number of cross-sectional studies have looked at the lifetime incidence of experiencing patient suicide among mental health workers in general and psychiatrists in particular. A national survey of 586 randomly selected psychiatrists in the USA (Chemtob et al, 1988) found that $51 \%$ of the 259 respondents had experienced at least one patient suicide in their career. Although there was no difference in terms of age or years of practice between those who had and had not experienced patient suicide, more postgraduate training was associated with a lower rate of patient suicide. The authors warn against overinterpretation of this result, and point out that higher-trained therapists may have less clinical contact with suicidal patients because of a shift towards administrative and supervisory duties. The probability that psychiatrists who experienced one patient suicide faced a second was $55 \%$ (i.e. about the same as the probability of experiencing just one).

Alexander et al (2000) sent a confidential coded postal questionnaire to 315 consultant psychiatrists in Scotland asking about the effect on them of patient suicide. Of the 247 psychiatrists who responded, $68 \%$ had experienced at least one such death. Of respondents who reported at least one patient suicide and provided details on the total number they had experienced, $21 \%$ had experienced one, $66 \%$ had experienced between two and six, and $13 \%$ had experienced between seven and fifteen. Respondents were also

Sharon Foley is a research registrar with the Dublin and East Treatment and Early Care Team (DETECT) service, an early detection and intervention pilot project for patients with psychosis in South Dublin and County Wicklow (DETECT Service, 1 Marine Terrace, Dun Laoghaire, County Dublin, Ireland. Tel.: +353 1236 6730; fax: +353 1236 6780; email: sharonrachelfoley@gmail.com). Her research interests include the epidemiology of psychosis and the development and effectiveness of early intervention services. Brendan Kelly is a senior lecturer in psychiatry in Dublin (Department of Adult Psychiatry, University College Dublin, Mater Misericordiae University Hospital, Ireland). His research interests include the epidemiology of psychosis and the relationship between mental illness and social factors. He was joint winner of the Gaskell Medal and Prize in 2003. 
asked about their 'most distressing suicide': in about $50 \%$ of cases this was the suicide of an out-patient, in $45 \%$ it was an in-patient and in $4 \%$ the individual had been a day patient.

Dewar et al (2000) approached 128 trainee psychiatrists in Scotland, 47\% of the 103 respondents reported the suicide of a patient who was under their care; the number of suicides experienced ranged from one to five. In addition to the $47 \%$ who reported direct experience of patient suicide, a further $40 \%$ had been involved in the consequences of suicide (e.g. the suicide of a patient who was not directly under their care). In a similar study, Courtenay \& Stephens (2001) sent a postal questionnaire to 400 trainee psychiatrists in the South Thames region of England. Of the 203 who responded, $54 \%$ reported experiencing at least one patient suicide; of this group, 39\% had experienced more than one, and the total number of suicides experienced ranged from one to seven. Yousaf et al (2002) contacted 89 psychiatric trainees in the UK and found that $43 \%$ of the 53 respondents had already experienced one or more patient suicides.

Overall, these studies indicate that about $50-70 \%$ of consultant psychiatrists and $40-50 \%$ of psychiatric trainees experience at least one patient suicide. In addition, a significant proportion experience more than one throughout the course of their careers. In the context of the broader community mental health team, Linke et al (2002) found that $86 \%$ of staff across various disciplines had experienced patient suicide; this higher rate most likely reflects a high level of shared case-load in the multidisciplinary setting of such teams.

\section{Effects on mental health workers}

Patient suicide can have significant personal and professional effects on mental health workers, including psychiatrists (Box 1).

Chemtob et al (1988) used Horowitz's Impact of Event Scale to assess the effect of patient suicide on psychiatrists in a national survey throughout the USA. Despite vigorous attempts to optimise response rates, only $46 \%$ of those who were sent the questionnaire responded; the authors suggest this may be due to the reluctance of psychiatrists to deal openly with such a sensitive topic. Of the ones who did reply, those who had experienced at least one patient suicide reported that it caused significant disturbances in both their personal and professional lives, and $53 \%$ reported Impact of Event Scale scores comparable to those of individuals who had suffered the death of a parent. There was a negative relationship between years of psychiatric practice and the impact of patient suicide: the longer the psychiatrist had
Box 1 Effects of patient suicide on trainee and consultant psychiatrists

Personal

- Stress

- Guilt

- Social withdrawal

- Reduction in self-esteem

- Disruption to relationships

- Irritability at home

- Poor sleep

- Low mood

- Decreased self-confidence

- Symptoms of post-traumatic stress disorder

- Feelings of anger, shame and isolation

Professional

- Fears of litigation and retribution

- Greater use of suicide observations

- More detailed note-keeping and communications

- Lower thresholds for using mental health legislation

- More defensive approaches to patient risk

- Self-doubt

- Consideration of early retirement

Based on Chemtob et al (1988), Gitlin (1999),

Alexander et al (2000), Dewar et al (2000),

Courtenay \& Stephens (2001), Yousaf et al (2002)

been in practice, the lower the reported levels of guilt, social withdrawal, reduction in self-esteem and disruption to relationships following patient suicide.

Alexander et al's (2000) study (mentioned above) found that $33 \%$ of the consultant psychiatrists whohad experienced at least one patient suicide reported an adverse impact on their personal lives, characterised by irritability at home, poor sleep, low mood and decreased self-confidence. Forty-two per cent also reported an impact on their professional practice, characterised by greater use of suicide observations, more-detailed note-keeping and communications, lower thresholds for using mental health legislation and more defensive approaches to patient risk. Fifteen per cent of consultants considered taking early retirement as a result of patient suicide.

Yousaf et al (2002) found that $52 \%$ of the psychiatric trainees in their study who had experienced patient suicide had signs of clinical stress in the aftermath of the event and $29 \%$ remained clinically stressed despite the passage of time. Courtenay \& Stephens (2001) found that $51 \%$ of their psychiatric trainees reported a moderate emotional impact after a patient suicide and $24 \%$ reported a severe emotional impact. 
Dewar et al (2000) likewise reported significant effects on psychiatric trainees following patient suicide: $31 \%$ reported an adverse effect on their personal lives and 39\% an adverse effect on their work. In addition, $9 \%$ considered a change of career in the aftermath of patient suicide.

Overall, these studies indicate that patient suicide has a significant effect on the personal and professional lives of consultant psychiatrists and psychiatric trainees. Gitlin (1999) provides a particularly valuable account of a psychiatrist's reaction to a patient's suicide, characterised by symptoms of post-traumatic stress disorder, feelings of anger, shame and isolation, and fears of litigation and retribution resulting in substantial self-doubt. Gitlin goes on to discuss the factors that come into play when a psychiatrist is faced with a patient suicide: the quality of the relationship between the doctor and patient, the psychiatrist's own psychological make-up, and his or her level of training. Clearly, the effects of patient suicide on mental health workers can be pronounced, prolonged and profound.

\section{Coping mechanisms}

As with any traumatic event, mental health workers use a range of different coping mechanisms in the aftermath of patient suicide. However, Chemtob et al (1988) point out that there is a dearth of established protocols to guide psychiatrists in this situation and a similar lack of formal teaching on the subject in most psychiatric training programmes. These authors also emphasise the reported benefits of consultation with colleagues and peer-group support in the aftermath of patient suicide (Chemtob et al, 1988).

Alexander et al (2000) asked consultant psychiatrists what helped them cope with patient suicide and found that the most effective support came from consultants' own families and friends, other team members and other psychiatrists. Critical incident reviews were generally viewed as helpful, whereas media coverage, legal proceedings and the prospect of litigation were significant causes of distress. Fifteen per cent of consultants had chosen to attend the patient's funeral and $63 \%$ of these reported finding it helpful. Alexander et al (2000) also asked psychiatrists if they found the patient's family helpful following a suicide. Of those for whom this question was applicable, some 36\% reported that the patient's family had been helpful, and $14 \%$ that the family had been very helpful.

Like consultant psychiatrists, psychiatric trainees also derive most support from informal processes involving family and colleagues: Dewar et al (2000), for example, report that psychiatric trainees derived most support from their consultants, team colleagues, family members and friends. Courtenay \& Stephens (2001) found that $46 \%$ of trainees who had experienced patient suicide expressed a need for help and the most common sources of help were their consultant and their community mental health team. Not all trainees who needed support, however, were able to access appropriate help: Courtenay \& Stephens found that $40 \%$ of trainees felt their need for help following patient suicide went ignored, and Dewar et al (2000) report that $6 \%$ of respondents who sought support from their consultants found them to be unhelpful.

Overall, these studies indicate that both consultant psychiatrists and psychiatric trainees derive most support from informal contacts with team members, family and friends. This reliance on informal sources may reflect a number of different factors, including the non-availability of formal support structures, difficulties availing of such facilities where they exist and/or the highly sensitive and personal nature of patient suicide.

\section{A pilot scheme for dealing with the issue of patient suicide}

In light of the relatively high incidence and prolonged effects of patient suicide in psychiatric practice, one of us (B. D. K.) undertook to develop a local initiative to address the issue at the level of the community mental health team. This initiative was informed by a literature review that clearly indicated, as outlined above, that: (a) the most valued support following patient suicide comes from contacts with consultants and other team members, as well as family and friends; and (b) many psychiatrists identify a need for training programmes and formal systems of support to complement informal support in addressing the issues raised by patient suicide (Chemtob et al, 1988; Gitlin, 1999; Dewar et al, 2000; Courtenay \& Stephens, 2001; Yousaf et al, 2002).

In particular, Alexander et al (2000) report that some consultants identified a need for patient suicide to be regarded as an inevitable rather than simply a possible event, so that staff could be better prepared for such an eventuality. In his account of one psychiatrist's reaction to patient suicide, Gitlin (1999) notes that death reflects the natural history of severe medical disorders such as heart failure and malignancies, and comments that the outcome of suicide in patients with severe depression may similarly reflect the natural history of the illness. While warning against adopting this philosophy in a nihilistic way, Gitlin goes on to discuss the personal benefits of developing a more objective outlook and avoiding a hypertrophied sense of responsibility over patients' lives. 
Following multidisciplinary team consultation, it was decided to have 'patient suicide meetings' at regular but infrequent intervals. The aim of these meetings would be to be to acknowledge the occurrence of patient suicide in psychiatric practice and explore its potential effects on community mental health teams. It was agreed that the meetings would be devoted to general issues regarding patient suicide and would not make detailed reference to specific patients. Meetings were to take place every 4 months, regardless of the occurrence or non-occurrence of a suicide in the intervening period. It was hoped that the very existence of such a programme of meetings would serve to acknowledge the high likelihood of patient suicide and to prepare the team for its likely effects on personal and professional lives.

All members of the multidisciplinary team were invited to the meetings, including the consultant psychiatrist, psychiatric trainees, community mental health nurses, nurses from the in-patient unit, administrative staff (e.g. secretary, receptionist) and other members of the multidisciplinary team. To provide some structure to the meetings and to minimise the likelihood that the discussion would focus on any specific patient, it was agreed to use a 'journal club' format. For the first meeting, a psychiatric trainee prepared and presented the questionnaire study of Alexander et al (2000) on the effect of patient suicide on consultant psychiatrists, and a community mental health nurse presented Linke et al's (2002) paper on the impact of suicide on community mental health teams.

It is hoped that this series of 'patient suicide meetings' will help the multidisciplinary team to acknowledge and address some of the issues related to patient suicide in a prospective fashion, independent of the circumstances of any specific patient and independent of the occurrence of any specific suicide. Although it will be difficult to evaluate the effectiveness of this initiative systematically, we hope to elicit feedback from team members as the process unfolds, and to modify and refine the process on the basis of experience in the coming years.

\section{Conclusions and recommendations}

For mental health workers, the effects of patient suicide can be pronounced, prolonged and profound. Indeed, the risk of patient suicide, even if it does not occur, may have a significant effect on morale: Deary et al (1996), for example, have shown that psychiatrists are more emotionally exhausted and have higher levels of depression than their medical and surgical colleagues. Patient suicide and the risk of patient suicide undoubtedly contribute to this exhaustion and depression among psychiatrists.

\section{Box 2 Recommendations}

- Greater emphasis on patient suicide during training

- Enhanced formal and informal support systems

- Increased awareness of the burden that informal support places on family and friends

- Exploration of other support (e.g. spiritual support, contact with the patient's family)

- Local team initiatives to identify potential issues and support

What can be done to lessen the adverse impact of patient suicide on psychiatrists? Some recommendations are summarised in Box 2. In the first instance, it is important to note that some degree of grieving is appropriate following the death of a patient; it is the prolonged negative effects on the personal and professional lives of psychiatrists that need to be addressed. Most studies on this topic identify a need for training programmes and formal systems of support in order to address the issues raised by patient suicide (Chemtob et al, 1988; Gitlin, 1999; Dewar et al, 2000; Courtenay \& Stephens, 2001; Yousaf et al, 2002). In their study of psychiatric trainees, Dewar et al (2000) reported that $28 \%$ of respondents had received some training in relation to patient suicide and most of these found it helpful in the long run.

Colleagues are consistently identified as an important source of support following patient suicide (Chemtob et al, 1988; Alexander et al, 2000; Dewar et al, 2000; Courtenay \& Stephens, 2001).

Hodelet \& Hughson (2001) provide practical advice on addressing the obligations and expectations of psychiatrists in the short and medium term following a patient suicide. They emphasise the role of dedicated team review following the death, possibly in the form of a 'suicide review conference' (Bartels, 1987) that looks at the death in the context of the psychological profile of the patient. They highlight that doctors in the UK who need more formal assistance can look to the British Medical Association, the Royal College of Psychiatrists and the National Counselling Service for Sick Doctors. Campbell \& Fahy (2002) also address the role of the doctor following a patient's suicide and point out that effects on the psychiatrist may be compounded by an obligation to address the needs of other team members (e.g. psychiatric trainees). They also emphasise the importance of appropriate support for the patient's family and an acknowledgement of the needs of other patients, particularly following suicide in an in-patient setting. 
Local team initiatives may also have a significant role to play in acknowledging the likelihood of patient suicide and prospectively identifying possible effects on the community mental health team. The nature and effectiveness of such initiatives may depend on the constitution of the team in question and members' previous experience of patient suicide.

Two final points merit particular attention. First, the patient's own family can have a wide variety of reactions to suicide, including hostility and denial (Hawton, 1986). None the less, as mentioned above, Alexander et al (2000) report that half of psychiatrists who responded to a question about the supportiveness of patients' families described the family as either helpful or very helpful. Appropriate engagement with the patient's family may therefore represent a significant source of support for both parties and may merit further exploration. Second, the literature consistently indicates that psychiatrists derive significant informal support from family and friends following patient suicide (Chemtob et al, 1988; Alexander et al, 2000; Dewar et al, 2000). This may represent a difficult, complex burden of support for family and friends - and one that merits acknowledgement and appreciation following resolution of the acute reaction to the death.

\section{Declaration of interest}

None.

\section{References}

Alexander, D., Klein, S., Gray, N., et al (2000) Suicide by patients: questionnaire study of its effect on consultant psychiatrists. $B M J, 320,1571-1574$.

Bartels, S. (1987) The aftermath of suicide on the psychiatric inpatient unit. General Hospital Psychiatry, 9, 189-197.

Campbell, C. \& Fahy, T. (2002) The role of the doctor when a patient commits suicide. Psychiatric Bulletin, 26, 44-49.

Chemtob, C., Hamada, R., Bauer, G., et al (1988) Patients' suicides: frequency and impact on psychiatrists. American Journal of Psychiatry, 145, 224-228.

Courtenay, K. P. \& Stephens, J. P. (2001) The experience of patient suicide among trainees in psychiatry. Psychiatric Bulletin, 25, $51-52$.

Deary, I., Agius, R. \& Sadler, A. (1996) Personality and stress in consultant psychiatrists. International Journal of Social Psychiatry, 42, 112-123.

Dewar, I. G., Eagles, J. M., Klein, S., et al (2000) Psychiatric trainees' experience of, and reactions to, patient suicide. Psychiatric Bulletin, 24, 20-23.

Gitlin, M. J. (1999) A psychiatrist's reaction to a patient's suicide. American Journal of Psychiatry, 156, 10.

Hawton, K. (1986) Aftermath of child and adolescent suicide. In Suicide and Attempted Suicide among Children and Adolescents, pp. 49-54. Sage Publications.

Hodelet, N. \& Hughson, M. (2001) What to do when a patient commits suicide. Psychiatric Bulletin, 25, 43-45.

Linke, S., Wojciak, J. \& Day, S. (2002) The impact of suicide on community mental health teams: findings and recommendations. Psychiatric Bulletin, 26, 50-52.

Yousaf, F., Hawthorne, M. \& Sedgwick, P. (2002) Impact of patient suicide on psychiatric trainees. Psychiatric Bulletin, 26, 53-55.

\section{MCQs}

1 Regarding patient suicide:

a patient suicide is a very uncommon event

b less than $50 \%$ of consultant psychiatrists lose a patient to suicide

c patient suicide affects all team members, not just psychiatrists

d few psychiatrists experience more than one patient suicide

e less than $30 \%$ of psychiatric trainees experience a patient suicide.

2 The effects of patient suicide:

a are greater for psychiatrists who have been in practice longer

$\mathrm{b}$ tend to be contained within the workplace

c tend to be minor

d include professional effects such as greater use of suicide observations

e tend to be short-lived.

3 Formal teaching about patient suicide during training:

a is commonly available for trainee psychiatrists

$\mathrm{b}$ is of little benefit

c aims to prospectively address issues related to patient suicide

d should be excluded from training programmes

e should be left until higher specialist training level.

4 The following are generally felt to be unhelpful following patient suicide:

a informal support from colleagues

b informal support from family and friends

c consultant psychiatrists

d spiritual support

e media coverage.

5 Future developments could usefully include:

a compulsory attendance of a team member at the patient's funeral

b formal incident review committees to apportion blame and accountability

c ignoring the issues associated with patient suicide until they actually occur

$\mathrm{d}$ developing local team initiatives in relation to patient suicide

e avoiding contact with the patient's family.

\section{MCQ answers}

$\begin{array}{llllllll}1 & & 2 & & 3 & & & 5 \\ \text { a F } & \text { a F } & \text { a F } & \text { a F } & \text { a F } \\ \text { b F } & \text { b F } & \text { b F } & \text { b F } & \text { b F } \\ \text { c T } & \text { c F } & \text { c } \text { T } & \text { c F } & \text { c F } \\ \text { d F } & \text { d T } & \text { d F } & \text { d F } & \text { d T } \\ \text { e F } & \text { e F } & \text { e F } & \text { e T } & \text { e F }\end{array}$

